\title{
La teoría de construcción de carrera y diseño de vida, las críticas que se le hacen y su posible aplicación para la orientación vocacional de grupos en América Latina, complementándola con la pedagogía crítica de Freire
}

\author{
Career Construction Theory and Life Design, the Criticisms that it Receives \\ and its Possible Applications to Vocational Guidance in Groups of Latin \\ America, Complementing it with Freire's Critical Pedagogy
}

Mauricio Navarro-Bulgarelli

Universidad de Costa Rica

San José, Costa Rica

mauricio.navarro@ucr.ac.cr

(iD) https://orcid.org/0000-0002-0762-3687

RECIBIDO: 7 de setiembre de 2021 / ACEPTADO: 16 de octubre de 2021

\section{Resumen}

En este ensayo se presentan los principales postulados de la teoría de construcción de carrera y el paradigma de diseño de vida propuestos por Mark L. Savickas. Además, se comentan varias de las críticas que recibe este enfoque. Por ejemplo, el hecho de que responde a una realidad contextual diferente a la latinoamericana, con una lógica de capital humano que fomenta la individualización y que a su vez se plantea para ser utilizada en orientación individual únicamente. Posteriormente, se propone una manera de aplicar los principales postulados del paradigma del diseño de vida a la orientación vocacional de grupos en contextos latinoamericanos. Para ello, al discurso narrativo que presenta este paradigma se puede complementar con el discurso crítico y emancipador de la pedagogía crítica de Paulo Freire. De esta forma, la perspectiva individualista que caracteriza al mundo posmoderno puede ser contrarrestada por medio de un discurso solidario y de transformación social. Además, el promover experiencias culturales que amplíen la cosmovisión de las personas estudiantes colabora a configurar narrativas vocacionales positivas que pueden favorecer la vivencia de vocaciones que busquen ir más allá de las realidades de injusticia social que caracterizan a las sociedades latinoamericanas. Para esto, se parte de una visión amplia de carrera que incluye no solo trayectorias educativas y ocupaciones laborales, sino además voluntariados, compromisos sociales y familiares que la persona desempeña a lo largo de su trayectoria vital.

Palabras clave: Teoría de construcción de carrera, paradigma del diseño de vida, pedagogía crítica, orientación vocacional, orientación grupal.

\section{Abstract}

This essay presents the main postulates of the Career Construction Theory and the Life Design Paradigm proposed by Mark L. Savickas. In addition, several of the criticisms that this approach receives are commented. For example, it 
responds to a contextual reality different from the one in Latin America. It also has a human capital logic that encourages individualization and that can be used only in individual counseling. Subsequently, a way to apply the main postulates of the Life Design Paradigm to the vocational guidance of groups in Latin American contexts is proposed. To achieve this, the narrative presented by this paradigm can be complemented by the critical and emancipatory approach of Paulo Freire's critical pedagogy. In this way, the individualistic perspective that characterizes the postmodern world can be countered through a discourse of solidarity and social transformation. In addition, promoting cultural experiences that expand the worldview of students helps to configure positive vocational narratives that can favor the experience of vocations that seek to go beyond the realities of social injustice that characterize Latin American societies. To do this, a broad career vision is considered, one that includes not only educational trajectories and work occupations, but also volunteering, social, and family commitments that the person performs throughout their life.

Key words: Career construction theory, life design paradigm, critical pedagogy, vocational guidance, group counseling.

\section{Introducción}

La construcción de carrera y diseño de vida (life design and career construction) es la teoría más citada en la última década en la investigación internacional relacionada con temas vocacionales (Juntunen, Motl y Rozzi, 2019). Desarrollada por Mark L. Savickas, principalmente en los primeros años de este siglo, el life design se plantea como una continuación de la evolución teórica que ha tenido la orientación vocacional, partiendo de los postulados iniciados cronológicamente por Parsons, y luego continuados por Holland y Super, entre otros. Esta teoría se presenta como un paradigma que responde a la sociedad actual de este siglo XXI, es decir, un mundo incierto, no lineal, no predecible, y siempre cambiante de la trayectoria de cada persona (Puertas, 2014).

Entre las críticas que algunas personas autoras le hacen a este postulado teórico, es que responde a una realidad norteamericana, y que no puede ser fácilmente aplicable a la realidad de países del sur del mundo, por ejemplo, en Latinoamérica, en donde la realidad contextual es muy diferente a la norteamericana (Hooley, 2021; McMahon, 2014; McMahon y Arthur, 2019). Además, esta teoría se plantea principalmente para una asesoría uno a uno, la cual es común en países del norte, por ejemplo, en los Estados Unidos de Norteamérica.

Sin embargo, aunque en Latinoamérica se da la orientación individual, muchas de las intervenciones de orientación son a nivel grupal y colectivo. Tal es el caso de Costa Rica, en donde la orientación vocacional se realiza, principalmente, en lecciones de orientación dentro de los centros educativos; las cuales en muchos casos están dentro del horario regular de las clases, especialmente en los colegios académicos de educación secundaria. En esta modalidad, se da una orientación grupal llamada colectiva, la cual es definida como "una interacción que se origina entre una persona profesional que orienta y un grupo de personas a orientar, reunidas de manera temporal en un espacio físico, o natural, tratando de comprender las circunstancias y exigencias del vivir” (Vargas, 2020, p. 135).

Desde esta realidad, a la persona profesional que labora en estos centros educativos le queda poco tiempo para poder atender de manera individual, y ciertamente no le es posible atender personalmente a la totalidad del estudiantado que tiene a su cargo. Por esta razón que se prioriza la orientación colectiva, ya que el trabajar con grupos se promueve una mayor cobertura de la población meta, así como por su carácter preventivo, lo que permite brindar la orientación colectiva como proceso continuo y sistemático con mayores alcances.

En este ensayo se pretende, por un lado, presentar los principales postulados de la teoría de construcción de carrera y el diseño de vida para su mayor comprensión. Y por otro, comentar algunas de las principales críticas que se le hacen a esta teoría para argumentar el por qué se considera que algunos de sus postulados teóricos podrían tomarse en cuenta en las intervenciones orientadoras a nivel grupal en 


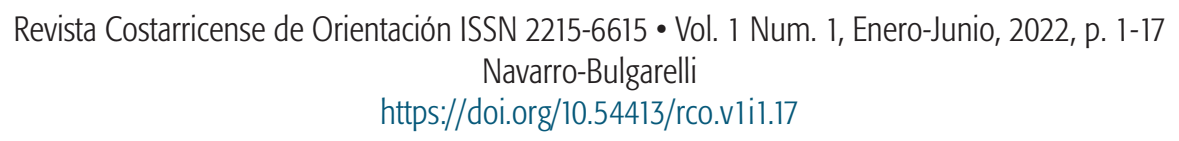

contextos latinoamericanos. Finalmente, se presenta una propuesta para hacerlo, en donde se contempla la teoría presentada por Savickas con aspectos teóricos propios de la pedagogía crítica de Paulo Freire.

Para lograr estos objetivos planteados, este ensayo consta de cuatro partes. Primero, se presentan las principales ideas de la teoría de construcción de carrera y diseño de vida. Posteriormente, se comentan algunas de las críticas que más se le han señalado a esta teoría. Luego se hace una breve introducción a la pedagogía crítica de Paulo Freire, y así se elabora una propuesta de intervención orientadora que pueda ser aplicable en grupos donde se desarrolle la orientación vocacional.

\section{Principales ideas de la teoría de la construcción de carrera y diseño de vida}

En este apartado se introducen los principales aspectos de la definición de la teoría de la construcción de carrera y su relación con las características del mundo posmoderno. También, se abarca el concepto del diseño de vida como el tercer paradigma de la orientación vocacional y sus características. Además, se presenta el concepto de carrera desde este paradigma y una breve referencia a los pasos para la intervención desde el diseño de vida.

\section{La teoría de la construcción de carrera y su relación con el mundo posmoderno}

La teoría de la construcción de carrera fue desarrollada por Mark L. Savickas durante la primera década del siglo XXI, y busca responderle a una sociedad posmoderna en donde, en palabras de este autor, hemos entrado en la era de los trabajadores inseguros que ya no están limitados por una sola organización o en el mismo trabajo durante tres décadas. El nuevo mercado laboral incluye una "economía de trabajos por encargo" que exige ver la carrera no como un compromiso de por vida con un solo ente empleador, sino como servicios de venta y una cartera de habilidades para una serie de entes empleadores que necesitan proyectos completados (Savickas, 2019).

La individualización se considera la característica central de las sociedades posmodernas, las cuales tienen trabajos des-estandarizados y patrones de ciclo de vida des-institucionalizados, en donde el curso de la vida ha hecho que la entrada en roles adultos sea más variable, menos estratificada por edades y usualmente requiriendo más tiempo. La vida se ha vuelto menos predecible, menos regulada, menos estable, y menos ordenada. Las estructuras sociales posmodernas hacen que sea difícil, e incluso imprudente, que las personas se comprometan con instituciones y organizaciones. En cambio, las personas se pueden comprometer con las trayectorias de vida elegidas por ellas mismas (Savickas, 2019).

Se espera que una persona en su trayectoria laboral ocupe no menos de diez trabajos diferentes, o mejor dicho asignaciones diferentes. Por esta razón, las personas deben hacerse empleables, el aprendizaje es de toda la vida, se debe trabajar en la inteligencia emocional y la adaptación rápida a los cambios. El mundo laboral actual requiere cada vez de más esfuerzo, más autoconocimiento y una gran confianza en sí misma. Todas estas características que describen a la sociedad actual hacen que las personas sufran de ansiedad e inseguridad. La intervención orientadora desde la teoría de construcción de carrera permite que la persona orientada forme, mantenga y reconstruya una narrativa de su identidad, por medio de la cual construye una historia de vida que es de su agrado, para así utilizarla en las continuas transiciones y cambios laborales que podrían ser traumáticos de otra forma. En la medida que una persona pasa de una labor a otra, deja atrás lo que hacía, pero no lo que es. El aferrarse a la propia historia de vida que se construye proporciona coherencia, continuidad y una forma de seguir viviendo una vida con propósito y sentido para la persona que así la asume, logrando así bajar sus niveles de ansiedad e inseguridad personal (Savickas, 2012). 


\section{El diseño de vida como el tercer paradigma de la orientación vocacional}

Por su parte, el diseño de vida (life design) se presenta como un paradigma que responde a la lógica de construcción de carrera, más que de ajuste o de desarrollo de carrera, siendo así el tercer paradigma de la orientación vocacional (Savickas, 2012).

Según este autor, en el primer paradigma de la orientación vocacional (vocational guidance) la perspectiva objetiva de las diferencias individuales ve a las personas como los actores de su vida, y se caracterizan según puntuaciones psicométricas en rasgos para así ajustarse en las ocupaciones que se asemejen mejor a dichas puntuaciones.

Por otro lado, el segundo paradigma (career education), desde una perspectiva subjetiva visualiza a las personas como los agentes de su vida, y se caracteriza por la formación en competencias para enfrentar las tareas del desarrollo esperadas, para de esa forma implementar nuevas actitudes, creencias y competencias en el desarrollo de sus carreras.

Finalmente, el tercer paradigma de la orientación vocacional es el del diseño de vida (life design), en donde desde una perspectiva del construccionismo social, ve a las personas orientadas como autores de su vida. Este paradigma se caracteriza por las narraciones de las historias autobiográficas, en donde se busca identificar los temas de vida recurrentes, por medio de los cuales las personas van construyendo sus carreras. De esta forma, las personas pueden analizar las posibilidades de su contexto, los procesos dinámicos de su vida, las progresiones no lineales, las múltiples perspectivas y los patrones personales que les ayudan a construir una carrera flexible, y tomar decisiones que los lleven a acciones acordes y comprometidas a la narrativa de su identidad (Savickas, 2012).

\section{Características del modelo del diseño de vida}

El modelo del diseño de vida se centra más en la pregunta de ¿cómo quieres ser?, en vez de la pregunta de ¿qué quieres ser? (Savickas, 2013). Otra pregunta relacionada que la persona debe hacerse desde esta visión es: ¿cómo debo diseñar mi vida para alcanzar eso que aspiro para mi vida? (Savickas, 2015). De esta forma, el diseño de vida conduce a métodos construccionistas que imponen significado al comportamiento vocacional (Savickas, 2019). Se parte de la premisa que las personas utilizan historias para organizar sus vidas, dar forma a sus identidades y darles sentido a sus problemas (Savickas, 2013).

El “yo", es decir el "sí mismo", se construye a partir de las palabras que la persona orientada escoge para formar sus autoconcepciones, y se sostiene al asumirse roles sociales que lo respalden dentro de diversos contextos, como podrían ser el lugar adonde se vive, los cursos de formación que se reciben, las labores ocupacionales y voluntariados que se asumen y la iglesia a la que se asiste. Todas estas acciones construyen una trama (plot) que no solo le da sentido y significado a la construcción que la persona está haciendo de su carrera, sino que guían las decisiones vocacionales que la persona toma y articulan una narrativa de su identidad, que los ayuda a auto-entenderse y orientarse en su mundo social (Puertas, 2014).

Es por eso por lo que, en las trayectorias de vida, las personas deben revisar constantemente su narrativa de la identidad, para adaptarla a las nuevas circunstancias y experiencias que su vida le está presentando, y así buscar integrar nuevas experiencias que sean significativas para esta trama de vida que construye su propia historia (Savickas, 2012). En pocas palabras, la identidad se construye a partir de la historia propia que nos narramos, y se reafirma en los roles sociales que asumimos para confirmar esa historia que nos creamos. 


\section{El concepto de carrera dentro de este paradigma}

En lo que respecta al concepto de carrera, esta es vista no como la suma de formación recibida y puestos laborales ocupados por una persona, sino más bien como el patrón/modelo de estas experiencias en un todo cohesivo/integrador, que produce una historia significativa, con propósito y sentido para la persona (Puertas, 2014). Esta definición de carrera se acerca más a lo que también se conoce como trayectorias de vida (Savickas, Nota, Rossier, Dauwalder, Duarte, Guichard, Soresi, Van Esbroeck y Van Vianen, 2009).

Este paradigma aborda cómo el mundo de la carrera se hace a través del construccionismo personal y social. Es decir, a partir de las representaciones que se van haciendo de la realidad, se va construyendo la carrera, impulsado por la adaptación a un entorno en lugar de por la maduración de las estructuras internas (Puertas, 2014).

En este modelo la carrera es la historia que la gente cuenta sobre su vida laboral. La narración autobiográfica proporciona un significado estabilizador para la guía interna y la autodirección durante los tiempos de transición. La historia fomenta el razonamiento biográfico que permite a una persona imponer unidad de propósito en las transiciones para convertir discordantes yuxtaposiciones en síntesis coherentes (Savickas, 2019). Es decir, las personas construyen sus carreras encontrando el significado a su comportamiento vocacional y a sus experiencias ocupacionales (Puertas, 2014).

\section{Los pasos para la intervención orientadora desde el diseño de vida}

Este paradigma es visto como un modelo de por vida, holístico, contextual y preventivo. Las intervenciones de orientación desde el diseño de vida tienen como objetivos aumentar la adaptabilidad, la narratividad y la actividad de las personas orientadas. La adaptabilidad tiene relación con el cambio, mientras que la narratividad aborda la continuidad. En conjunto, la adaptabilidad y la narratividad proporcionan a los individuos la flexibilidad y fidelidad de sí mismos que les permite involucrarse en actividades significativas para sus vidas de manera intencionada (Savickas et al., 2009).

A continuación se presentan, de manera muy general, los cinco momentos de la intervención orientadora planteados por el autor. Para las personas interesadas en profundizar en ellos, el autor puso a disposición de manera gratuita y en digital un documento llamado un Manual para la orientación desde el diseño de vida (Life-Design Counseling Manual). Desde su perspectiva, los pasos de este manual no buscan predecir, experimentar o medir científicamente la práctica orientadora. Sino más bien, desde su visión, es la práctica orientadora la que produce el discurso disciplinario y no que la orientación sea un producto del discurso (Savickas, 2015).

Las intervenciones desde este paradigma plantean una estructura en cinco momentos, que según Savickas $(2012,2015)$ son:

a) Construcción: Las personas orientadas llegan en busca de orientación debido a un incidente que los descoloca de su historia actual. Entonces la intervención empieza con la narración de este episodio, así como de la respuesta a la pregunta que la persona profesional en orientación le hace: "¿cómo puedo ser útil para ti hoy? Y luego, ¿hay algo más que pueda hacer por ti?, planteando así las metas esperadas del proceso en conjunto. Luego se construye la carrera de la persona orientada a partir de pequeñas historias que narra. Para ello se utiliza la entrevista de la construcción de carrera (career construction interview). 
b) Deconstrucción: de esas pequeñas historias, elaborada en conjunto profesional de la orientaciónpersona orientada, en búsqueda de ideas auto limitantes, prejuicios de roles y barreras culturales. Se analizan además posibles sesgos en las historias por género, raza o estrato social. Para ello se pueden discutir posibles omisiones en la historia o aspectos que parecen no tener un sentido claro al momento de ser narrados. Lo que se busca no es destruir la historia, sino más bien cuestionar posibles prejuicios en el pensamiento de la persona orientada, para así poder abrirse a la posibilidad de nuevos significados y conocimientos que puedan brindar posteriormente nuevas iniciativas para su diseño de vida.

c) Reconstrucción: de las historias dentro de una narrativa de la identidad que calce en un "retrato de vida" (lifeportrait) propuesto inicialmente por el profesional de orientación a la persona orientada para su valoración. La persona profesional en orientación intenta reconstruir las pequeñas historias en una gran historia, reuniendo las micro narraciones sobre incidentes importantes, episodios recurrentes, figuras significativas, momentos de autodefensa y experiencias que han cambiado la vida, en busca de los hilos de la historia, para tejer en un tapiz que refleje un sentido unificado de individualidad. Cuando las personas enfrentan desafíos e interrupciones, el patrón recurrente en el tema macro narrativo dirige, regula y sostiene sus acciones.

d) Co-construcción: de esta narrativa de la identidad en conjunto profesional de la orientaciónpersona orientada que permita visualizar las futuras acciones. Esta co-construcción incluye la trama ocupacional, el tema de la carrera y el arco del personaje. La idea es que la persona orientada pueda clarificarse, definiendo sus prioridades y, con una nueva visión de sí misma, pueda reorganizar sus sistemas de significados y empezar a ver cuál sería el siguiente episodio en su historia de carrera.

e) Acciones a tomar: en el nuevo episodio a escribir en la vida real de la persona orientada. Al final esta historia de carrera que ha sido co-construida en el proceso, les ayuda a las personas orientadas a vivir sus transiciones de carrera, manteniendo su ser esencial de manera intencionada, mediante la narrativa de su identidad. El sentido que las personas le den a su pasado les ayuda a enfrentar las transiciones en su presente, y los llevará a construir su futuro. El objetivo final del proceso es la toma de acciones concretas que sigan construyendo esta carrera de la persona orientada.

Para resumir el proceso de intervención, se podrían usar las palabras tensión, atención, intención y extensión para explicar a las personas orientadas el resultado de la intervención orientadora. La persona trajo cierta tensión para abordar en consulta con la persona profesional en orientación. Juntas, las dos personas prestan atención a esa tensión en forma de historias de carrera. Luego reconstruyen la tensión en la intención. Tras el proceso orientador, la persona orientada busca cierta extensión del yo en una acción intencional que resuelve la tensión (Savickas, 2012).

\section{Comentario a las críticas que se le hacen al paradigma del diseño de vida}

A continuación se presentan y comentan al menos cinco críticas que se le hacen al paradigma del diseño de vida, a saber: la necesidad de un entrenamiento psicológico específico recibido por la persona orientadora, que es una teoría dirigida únicamente a clase media blanca occidental con posibilidades de elección, su relación con la teoría del capital humano, el léxico que se utiliza y el hecho de que solo pueda ser aplicado en atenciones de orientación vocacional a nivel individual.

La primera crítica es mencionada por el mismo Mark L. Savickas, quien dice que a su teoría la han criticado debido a que, supuestamente, el facilitador del proceso debe tener un entrenamiento psicológico 


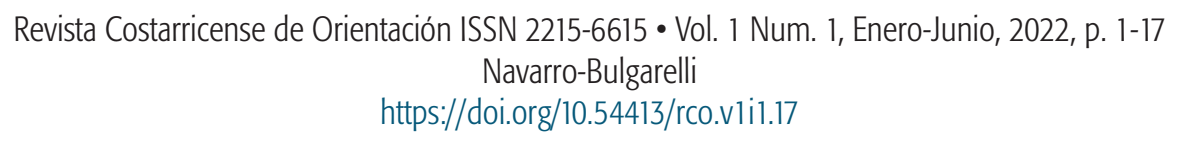

específico para poder interpretar las respuestas de la persona orientada, A esta crítica el mismo Savickas (2015) responde que no se trata de interpretar lo que la persona orientada narra, sino más bien de construir significado a partir de lo que narra. Es decir, no es una identificación de contenido latente y oculto dentro de un marco pre-existente de significados (como podría ser interpretar), sino más bien es una construcción de la persona orientada a partir de su descripción de los eventos vividos y sus perspectivas sobre ellos, al cual le darán un significado desde su propia narrativa de la identidad. En la perspectiva de este autor, la persona profesional solo acompaña este proceso personal (Savickas, 2015).

Sobre este mismo tema, otro autor menciona que, para evitar confundirse con las complejidades y contradicciones de la narrativa de una persona orientada, las personas profesionales de la orientación no se centran tanto en escuchar "los hechos", sino el "pegamento" que mantiene unidos los hechos mientras tratan de escuchar el tema que conforma la totalidad de la vida de la persona orientada. Organizar las acciones e incidentes, aparentemente aleatorios, reportados en las historias de carrera, dentro de una trama, se puede hacer de muchas formas. El profesional de la orientación escucha la "esencia" de las historias que cuenta la persona. La construcción de carrera implica convertir una preocupación personal en una ocupación pública. Mientras se escucha a una persona narrar sus historias, hay que concentrarse en identificar y entender su visión de mundo, para convertir la esencia en interés, la tensión en la intención y la obsesión en la profesión (Puertas, 2014).

$\mathrm{Al}$ respecto se considera que, con la formación recibida en la disciplina de Orientación a nivel de grado universitario, y en algunos casos incluso de posgrado universitario, además de la experiencia adquirida en el ejercicio de la profesión acompañando a las personas en la construcción de sus proyectos de vida y toma de decisiones vocacionales, la persona profesional en orientación tiene las bases suficientes para poder incorporar el diseño de vida y la teoría de construcción de carrera dentro de sus intervenciones profesionales. Lo que debe realizar la persona interesada en utilizar este modelo vocacional, es estudiar los principales fundamentos teóricos que presenta este discurso narrativo, y ponerlos en práctica desde su quehacer y con la creatividad profesional que le caracteriza. Es importante además que se empiecen a sistematizar y publicar las intervenciones profesionales, para así ir enriqueciendo cada vez más el bagaje teórico y metodológico de la orientación vocacional con ejemplos propios de nuestros contextos latinoamericanos.

Otra de las críticas que se le hacen, no solamente a esta teoría en particular, sino que a las teorías del desarrollo y construcción de carrera en general, es el hecho de que están dirigidas únicamente a una clase media blanca occidental con posibilidades de elección (McMahon, 2014).

Relacionado con lo anterior, se señala también que es una teoría muy occidental, escrita en inglés, desde una realidad "del norte", que asume una cultura individualista. Presupone que las personas tienen siempre opciones para poder elegir y parten de la premisa que las personas desean utilizar sus carreras como un medio para lograr la autorrealización. Además, se menciona que en su mayoría las teorías asumen apoyo tácito a la agenda neoliberal, enfatizando la agencia individual a expensas del cambio estructural (McMahon y Arthur, 2019).

$\mathrm{Al}$ respecto se considera que, si bien es cierto en otros contextos como los latinoamericanos, las posibilidades de elección no siempre son tan amplias como podrían ser en el contexto anglosajón del que procede esta teoría, la centralidad del discurso narrativo del paradigma del diseño de vida puede ser aplicado a los contextos latinoamericanos. Los conceptos de narratividad, identidad narrativa, los pasos de intervención del diseño de vida, la flexibilidad que se debe tener en la construcción de la carrera y la importancia de mantenerse empleable en el contexto laboral incierto y cambiante que también caracteriza a los mercados latinoamericanos, son algunos de los ejemplos que presenta este paradigma que son aplicables para nuestros países del sur del mundo. 
Sin embargo, se considera fundamental para una aplicación de este modelo paradigmático en Latinoamérica el hecho de que al discurso narrativo de la construcción de carrera se le pueda complementar con un discurso crítico y emancipador, que busque sensibilizar a las personas orientadas en cuanto a las situaciones sociales, ambientales y de diversa índole que se dan en los contextos en los cuales se desenvuelven, y de esta forma pueda orientar la construcción de sus carreras personales no solo hacia el mejoramiento del bienestar personal, sino también, y necesariamente, hacia la búsqueda de un bien común. Esta idea parte de la postura del gran pedagogo latinoamericano Paulo Freire, quien sostenía que, por medio de la educación, el ser humano podía, en una primera instancia tomar consciencia de la situación que vive el mundo, para luego apropiarse de ella, e intentar transformarla, no desde el individualismo, sino desde la comunión y la solidaridad (Freire, 1970). Esta idea se ampliará más adelante en este ensayo.

Otro aspecto importante que se debe considerar para las intervenciones de orientación vocacional desde este paradigma en contextos latinoamericanos, es la idea introducida por Cunti y Priore (2020), quienes hablan sobre el cómo desde la educación, la orientación puede facilitar experiencias culturales que permitan ampliar el horizonte de las personas estudiantes, y que de esta manera pueda considerar en sus narrativas vocacionales nuevas alternativas que, de no vivir estas experiencias culturales facilitadas por el centro educativo, quizás no podría tomar en cuenta dentro de su narrativa de la identidad.

Según estas autoras, las diversas experiencias culturales y educativas que las personas estudiantes puedan vivir en la escuela, junto con la influencia positiva que puedan recibir de sus profesores y profesoras, contribuirán a configurar narrativas presentes y de futuro que puedan ir más allá del estrecho destino que podrían tener dados sus contextos sociales y familiares. De esta forma se pueden reconstruir identidades y devolver el derecho a la autodeterminación, garantizando la pluralidad de opciones para sus decisiones vocacionales (Cunti y Priore, 2020).

Una tercera crítica que se le hace, en general, a las teorías de desarrollo y construcción de carrera es su relación con la teoría del capital humano. Desde esta visión, la buena educación se define como un curso que conduce al individuo hacia un mejor salario, y una buena carrera es aquella en la que el individuo puede atraer un salario alto. Los esfuerzos humanos y la búsqueda de la vida, la libertad y la felicidad se reducen a ingresos en un balance financiero. Desde este punto de vista, la construcción de carrera se ve como un proceso de decisiones racionales de "inversión personal", cuando más bien el proceso de decisiones no es racional, ni lineal, sino que está influenciado por acciones, eventos y circunstancias de las cuales el individuo no tiene control (Hooley, 2021).

De esta forma, se critica que este tipo de acercamientos teóricos les dan a las personas total responsabilidad sobre sus decisiones en cuanto a las trayectorias educativas que toman, y la "inversión" que realizan en sí mismas sobre sus conocimientos y habilidades dentro de un modelo de "aprendizaje durante toda la vida" (lifelong learning). Es decir, se ignoran factores sociales y políticos que influyen y en muchos casos limitan considerablemente las alternativas de decisión en las trayectorias educativas que las personas pueden tomar. El autor argumenta que en muchos casos las alternativas educativas que permiten acceder a posiciones de bienestar, poder y oportunidades, son reservadas para un grupo privilegiado que puede acceder a ellas. Y el tratar de "predicar" que las decisiones son total responsabilidad del individuo ayuda a ocultar estas inequidades sociales y políticas que existen dentro de la sociedad (Hooley, 2021).

El énfasis que estas teorías dan a las narrativas individualizadas produce como consecuencia una ceguera a los análisis sociales. Tal enfoque en el individuo puede parecer que convierte al profesional en orientación vocacional en un actor apolítico que solo busca ayudar a las personas a promover sus propios 
intereses. Pero este autor sostiene que los servicios de orientación vocacional que se dan desde una visión de desarrollo y construcción de carrera han sido incorporados a la ideología de la teoría del capital humano, y este mismo enfoque en el individuo actúa como una herramienta mediante la cual se pide a las personas que compren los discursos de la meritocracia y el individualismo (Hooley, 2021).

Como respuesta a esta tendencia recién planteada, se concuerda con la propuesta del mismo autor, quien menciona que desde la orientación vocacional no solo se promueva el desarrollo y construcción de la carrera, sino también el desarrollo de una racionalidad emancipadora dentro de la carrera, para así poder educar en los individuos valores cívicos y pensamientos críticos que busquen no solo el propio bienestar, sino también el florecimiento de la humanidad, contribuyendo a un cambio social. El trabajo de la orientación vocacional debería ayudar a las personas a desarrollar una comprensión crítica del mundo, nombrando las diversas formas de opresión, problematizando normas, supuestos y relaciones de poder en busca de la construcción de un mundo más solidario y de acción colectiva (Hooley, 2021).

De esta forma, la orientación vocacional alcanzará uno de los objetivos de las teorías de desarrollo y construcción de carrera, el cual busca que la persona pueda responsabilizarse por la construcción de su propia carrera. Pero, además, permitirá que la persona se haga consciente de su propia realidad social y la de tantos otros a su alrededor, para que así se pueda responsabilizar del cambio social deseado y necesario al que puede aportar con sus decisiones vocacionales. Para ello, se puede complementar la base teórica que nos brinda la construcción de carrera con la pedagogía crítica y emancipadora de Paulo Freire (1970, 2004), idea que se desarrolla más adelante en este mismo ensayo.

Una cuarta crítica que el mismo Hooley (2021) introduce es la del lenguaje que se utiliza en este tipo de teorías. Conceptos como los de "cliente" deben repensarse, dada su relación con principios neoliberalistas y basados en la visión del ser humano como un capital. Es por eso por lo que el autor sugiere la pregunta: ¿somos humanos o somos capital?

Se concuerda con esta visión, dado que es importante que la persona profesional en orientación sea consciente del léxico que suele utilizar en sus intervenciones, ya que este responde a posiciones políticas que se sustentan con el uso de ciertas palabras. Por eso se propone que en América Latina se continúe utilizando el término "persona orientada”, en vez del concepto de "cliente”, más común en la literatura anglosajona. Pero, además, se propone incorporar conscientemente al léxico orientador, palabras como solidaridad, esperanza, libertad, justicia social, comunión, ternura y otras similares, las cuales son propias de una lógica de pedagogía crítica de Paulo Freire (1970, 2004).

Según a lo anterior, autores como Hyslop-Margison y Ayaz (2007), basados en el pensamiento de Freire, sostienen que el currículo escolar fundamentado en principios neoliberalistas desde la teoría del capital humano reduce la orientación vocacional y la educación para la carrera como una preparación para la adaptación al mercado laboral existente, y aceptando condiciones inestables y muchas veces indignas de empleo. Estos autores sostienen además que la agenda educativa neoliberalista no permite que en las instituciones educativas se hable de las injusticias sociales y de la transformación personal y social necesaria para mejorar las condiciones de vida de las personas. En su lugar, ellos proponen una educación vocacional que reflexione en la comprensión de los fenómenos sociales, para empoderar a las personas estudiantes y que estos se sientan sujetos activos de la historia, con el poder de transformarla para la búsqueda de más libertad y justicia social. A su vez, ellos sostienen que en las clases de secundaria las personas estudiantes deben ser empoderadas para que se pregunten sobre los problemas que afectan la vida social y laboral de las personas, analizando las causas estructurales de estos problemas y buscando soluciones que los incluya como agentes sociales de transformación (Hyslop-Margison y Ayaz, 2007). 
Finalmente, una de las características de la teoría de la construcción de carrera, es que se plantea para ser utilizada en la atención individual, en una relación uno a uno entre la persona orientada y la persona profesional en orientación, como lo explica Mark L. Savickas en su Manual para la orientación desde el diseño de vida (Savickas, 2015). Esto se puede criticar, principalmente, porque en muchos países la orientación vocacional se da mayoritariamente desde la atención a grupos de educación secundaria, por ejemplo, en Costa Rica, en donde las personas profesionales en orientación trabajan mayoritariamente desde la modalidad colectiva. En este país, en los colegios académicos la persona profesional en orientación cuenta con al menos una lección semanal con los grupos con los cuales trabajan sesiones colectivas. También se puede dar la atención a grupos por medio de talleres o atenciones personal-grupales. La atención individual, si bien se puede dar en los centros educativos, no se da con la totalidad del estudiantado que la persona profesional en orientación tiene a su cargo.

Por lo que limitar la orientación vocacional al uso de esta teoría como la plantea el autor (en modalidad individual), no permitiría abarcar a la totalidad de la población estudiantil, ya que la orientación individual es una "relación con carácter educativo, de asesoramiento y asistencial, que se origina de un encuentro cara a cara entre una persona profesional en este campo y otra con necesidad de ser orientada. La estrategia metodológica que se utiliza es la entrevista...” (Vargas, 2020, p. 120). Dada la gran cantidad de estudiantes que tiene que atender una sola persona profesional en Orientación, es humanamente imposible que la persona profesional le aplique una entrevista individual de construcción de carrera a la totalidad de la población estudiantil a la que atiende.

No obstante, se considera que algunos de los postulados teóricos y metodológicos que tiene el modelo de intervención desde el diseño de vida, se pueden aplicar en los procesos de orientación grupal; ya sea en sesiones colectivas, talleres o bien sesiones de personal grupal. Con las intervenciones grupales, el profesional puede orientar a la totalidad de la población estudiantil a su cargo por medio del paradigma del diseño de vida.

De esta manera, la esencia del discurso narrativo del que habla Savickas (2015), puede dar una base teórica y metodológica para los procesos grupales de intervención en orientación vocacional. A continuación se introduce brevemente la pedagogía crítica de Paulo Freire, la cual ayudará a explicar más adelante la intervención grupal que propone este ensayo.

\section{Breve introducción a la pedagogía crítica}

Es importante aclarar que los escritos de Paulo Freire hablan de la educación en general y no, específicamente, sobre la persona profesional en orientación. Para efectos de este ensayo, se parte del hecho de que la persona profesional en orientación es una persona educadora, y por tanto cuando se comenta o cita alguna idea de este autor la intervención orientadora está incluida en ella.

La propuesta de Paulo Freire (1970) parte de la premisa de que la educación será liberadora en cuanto sea creadora de criticidad, ya que esto compromete a las personas en el esfuerzo por transformar la realidad concreta y objetiva de la que se están concientizando. En la pedagogía crítica se busca que las personas se inserten en la realidad a fin de conocerla mejor, y así transformarla mejor.

Es así como la pedagogía crítica busca restaurar la humanidad tanto del oprimido como del opresor. Esto se logra superando la situación opresora, lo cual "implica el reconocimiento crítico de la razón de esta situación, a fin de lograr, a través de una acción transformadora que incida sobre la realidad, la instauración 
de una situación diferente, que posibilite la búsqueda del ser más” (Freire, 1970, p. 28). Este autor amplía su postura al afirmar que se procura "la superación de la contradicción opresores-oprimidos que, en última instancia, es la liberación de todos" (Freire, 1970, p. 29). Se propone la solidaridad como la manera de luchar juntos para la transformación de la realidad objetiva mediante la lógica del ser para otros.

Esta vocación humanizante se afirma en el ansia de libertad, de justicia, de lucha de los oprimidos por la recuperación de su humanidad despojada (Freire, 1970, p. 24). La pedagogía crítica "sirve a la liberación, se asiente en el acto creador y estimula la reflexión y acción verdaderas de los hombres sobre la realidad, responde a su vocación como seres que no pueden autenticarse al margen de la búsqueda y de la transformación creadora” (Freire, 1970, p. 65). Lo que se busca es que el ser humano tome consciencia de la situación del mundo en el que vive, y se "apropian de ella como realidad histórica y, como tal, capaz de ser transformada por ellos" (Freire, 1970, p. 67). Este autor latinoamericano recalca que "esta búsqueda del ser más no puede realizarse en el aislamiento, en el individualismo, sino en la comunión, en la solidaridad de los que existen” (Freire, 1970, p. 67). Desde su visión pedagógica, la educación verdadera es praxis, reflexión y acción del hombre sobre el mundo para transformarlo (Freire, 2004).

Desde esta propuesta, las personas que el autor llama oprimidos no deben entender la realidad actual como la única posibilidad, "sino como una situación que sólo los limita y que ellos pueden transformar. Es fundamental entonces que, al reconocer el límite que la realidad opresora les impone, tengan, en este reconocimiento, el motor de su acción liberadora" (Freire, 1970, p. 29). Desde este autor, el reconocimiento de la realidad debe llevar a una inserción crítica en ella, lo que a su vez conduce a una acción para transformarla.

Por otro lado, las personas que el autor llama opresores deben hacerse conscientes de que pueden estar siendo "herederos de la explotación o espectadores indiferentes de la misma" (Freire, 1970, p. 41). También, deben reconocer, para luego superar, la lógica de la opresión desde la cual "el valor máximo radica en el tener más y cada vez más, a costa, inclusive del hecho del tener menos o simplemente no tener nada de los oprimidos" (Freire, 1970, p. 39). Esta deshumanización, en palabras del autor, "no se verifica sólo en aquellos que fueron despojados de su humanidad, sino también, aunque de manera diferente, en los que a ellos despojan, en distorsión de la vocación de ser más” (Freire, 1970, p. 25). Según este autor, la violencia de los que él llama los opresores los despoja de su humanidad, instaurándoles el ser menos, en vez de esta vocación al ser más.

La pedagogía crítica de Paulo Freire enfatiza en la importancia del diálogo crítico y liberador, el cual supone la acción. Para ello, se propone la reflexión sobre la vocación del ser humano, que es hacia la humanización. Este diálogo reflexivo y crítico lleva a identificar las condiciones que niegan esta vocación de la humanización; es decir, la injusticia, explotación, opresión y en la violencia de los que él llama los opresores. Reflexionar sobre estas condiciones despierta esa vocación humana por la libertad, la justicia y la lucha de los oprimidos por la recuperación de esta humanidad despojada.

La consciencia crítica de la que habla este autor incluye la capacidad de analizar críticamente y desafiar las condiciones sociales opresoras que contribuyen a las iniquidades educativas, económicas y sociales. Mediante la pedagogía crítica, las personas que participan en los grupos pueden aumentar su consciencia de las estructuras sociales de opresión, desarrollar un sentido de comunidad y solidaridad con los otros, y obtener la motivación para comprometerse con la transformación de estas condiciones sociales.

Algunos autores recomiendan incluir la pedagogía crítica en los procesos de orientación vocacional, ya que esta tiene el potencial de reducir la discriminación mediante la motivación hacia el compromiso y la responsabilidad social. Estos autores también señalan la importancia de que cada persona se haga consciente 
de su propio rol dentro del sistema opresor, así como de las causas de las desigualdades sociales, para de esta forma disipar estereotipos sociales, superar la lógica del opresor-oprimido y desarrollar un compromiso hacia la construcción de un mundo más justo (Kenny, Blustein, Liang, Klein y Etchie, 2019).

Otro aspecto en el cual se enfatiza, desde la pedagogía crítica, es el de la educación problematizadora y liberadora, la cual busca despertar la consciencia mediante la comunicación. Es decir, la persona educadora, más allá de "depositar, de narrar, de transferir o de trasmitir conocimientos y valores a los educandos, meros pacientes, como lo hace la educación bancaria” (Freire, 1970, p. 61), dialoga con las personas estudiantes, para juntos educarse en comunión. Para este autor, nadie educa a nadie, ni nadie se educa solo. Entonces, el diálogo crítico permite que se dé la educación de todas las personas, siendo lo que él llama el mundo como mediador. Es decir, esos temas de la realidad mundial de los que se problematiza y dialoga son los que permiten que el aprendizaje sea un desafío consciente para transformar la realidad. Lo que realiza el educador es problematizar constantemente con las personas educandas sobre los temas a trabajar, y estos a su vez "se transforman ahora en investigadores críticos en diálogo con el educador, quien a su vez es también un investigador crítico” (Freire, 1970, p. 62). Es así como este autor plantea que:

Cuanto más se problematizan los educandos, como seres en el mundo y con el mundo, se sentirán mayormente desafiados. Tanto más desafiados cuanto más obligados se vean a responder al desafío. Desafiados, comprenden el desafío en la propia acción de captarlo. Sin embargo, precisamente porque captan el desafío como un problema en sus conexiones con otros, en un plano de totalidad y no como algo petrificado, la comprensión resultante tiende a tornarse crecientemente crítica, y por esto, cada vez más desafiada. A través de ella, que provoca nuevas comprensiones de nuevos desafíos, que van surgiendo en el proceso de respuesta, se van reconociendo más y más como compromiso. Es así como se da el reconocimiento que compromete. (Freire, 1970, p. 63)

Los procesos grupales de orientación vocacional pueden retomar esta idea del compromiso que despierta en las personas orientadas los desafíos sociales que se descubren mediantela aplicación de la pedagogía critica en las sesiones grupales. A continuación, se presenta la propuesta de intervención orientadora con grupos, tomando en cuenta las principales posturas analizadas hasta el momento en este ensayo.

\section{Propuesta de intervención orientadora con grupos}

Como se ha planteado, anteriormente, la persona profesional en Orientación puede agregarle al discurso narrativo de la construcción de carrera (el cual tiende a ser individualista) un discurso crítico y emancipador, que busque sensibilizar a las personas orientadas en cuanto a las situaciones sociales, ambientales y de diversas índoles que se dan en el mundo, y del cómo la construcción de la carrera personal no sólo debe buscar el mejoramiento del bienestar personal, sino también, y necesariamente, la búsqueda de un bien común; mediante el cual cada persona desde su propia carrera pueda aportar en la construcción de un mundo mejor, más bello, más solidario, más justo.

En seguida se presentan las preguntas que pueden guiar las sesiones grupales de orientación vocacional y luego la metodología planteada para este proceso grupal.

\section{Preguntas que guían las sesiones grupales}

La metodología que se explicará, más adelante, parte de una o dos preguntas guía por sesión, las cuales buscan que las personas orientadas vayan creando su narrativa vocacional. Estas preguntas surgen de 
las dos posturas teóricas analizadas en este ensayo; es decir, la teoría de construcción de carrera y su paradigma del diseño de vida junto con la pedagogía crítica.

Las preguntas propuestas se exponen en la siguiente tabla:

\section{Tabla 1}

Posibles preguntas por utilizar desde la construcción de carrera y la pedagogía crítica

\begin{tabular}{|c|c|}
\hline Preguntas basadas en la teoría de construcción de carrera & Preguntas basadas en la pedagogía crítica \\
\hline - Cuéntame de tu película o libro favorito en la infancia. & $\begin{array}{l}\text { - ¿Qué realidades de injusticia social o necesidades de la } \\
\text { humanidad te inquietan o te preocupan, y te gustaría aportar } \\
\text { algo para su solución? }\end{array}$ \\
\hline $\begin{array}{l}\text { - ¿Quiénes eran tus héroes/heroínas de infancia? } \\
\text { - Cuéntame de tres personas (diferentes de tu madre y padre) que } \\
\text { admirabas cuando eras niño o niña. Pueden ser personas reales, } \\
\text { personajes de caricaturas, superhéroes o personajes de libros }\end{array}$ & $\begin{array}{l}\text { - ¿Cuáles eran las causas sociales que estas personas que } \\
\text { admirabas defendían? ¿Cómo hacían esas personas de este un } \\
\text { mundo mejor? }\end{array}$ \\
\hline - ¿Cómo quieres ser como persona? & $\begin{array}{l}\text { - ¿De qué manera, con tu vida, puedes aportar en la construcción } \\
\text { de un mundo mejor, más justo, más bello, más solidario? }\end{array}$ \\
\hline - ¿Cómo debes diseñar tu vida para alcanzar lo que aspiras? & $\begin{array}{l}\text { - ¿Qué acciones solidarias puedes realizar para aportar a la } \\
\text { transformación de la causa social que te mueve? }\end{array}$ \\
\hline
\end{tabular}

Nota. Elaboración propia basado en Freire (1970, 2004), Kenny et al, (2019), Hyslop-Margison y Ayaz (2007), Savickas (2015).

Desde la metodología que se propone, se puede emplear solo una pregunta por sesión, o bien utilizar las dos preguntas de la misma fila en una sesión, es decir la pregunta de la izquierda (basada en la teoría de construcción de carrera) en conjunto con la pregunta de la derecha en la misma fila (basada en la pedagogía crítica). Cabe aclarar que estas preguntas son flexibles y pueden ser modificadas por la persona profesional en orientación. Las mismas son solo una guía para la aplicación de un proceso de orientación vocacional desde las ideas desarrolladas en este ensayo.

A continuación se explica la metodología del proceso de orientación grupal que se propone realizar con estas preguntas como guía.

\section{Metodología del proceso}

Se propone una metodología de sesiones colectivas en tres momentos: uno de producción individual, otro de diálogo grupal y el último de reflexión personal.

El primer momento de trabajo individual consiste en que cada participante elabore por escrito sus narraciones sobre la pregunta planteada para esa sesión en un portafolio de trabajo; el cual según Danielson y Abrutyn (1999), es una colección deliberada de los trabajos realizados por las personas estudiantes, orientada por los objetivos de aprendizaje que se persiguen. Esta herramienta pedagógica da la oportunidad de que tanto la persona estudiante como la persona docente (y en el caso particular del que estamos hablando la persona profesional en Orientación) comenten y reflexionen sobre el trabajo presentado en el portafolio, el cual queda como una evidencia del avance y aprendizaje adquiridos (Danielson y Abrutyn, 1999). 
La persona profesional en Orientación, como facilitador del proceso, puede guiar y acompañar a las personas participantes para que estas puedan alcanzar algunos de los objetivos del modelo de diseño de vida, los cuales tienen relación con identificar, por medio de las proyecciones que realiza en las respuestas a las preguntas guía, los temas de vida recurrentes en su narrativa de la identidad, así como las progresiones no lineales que han seguido en su trayectoria de vida, para luego poder analizar las posibilidades de su contexto que les ayudan a construir una carrera flexible y acorde a su narrativa de la identidad construida (Puertas, 2014).

En un segundo momento de la sesión se pasa a un diálogo grupal, el cual inicia compartiendo las respuestas individuales a la pregunta guía de la sesión de manera voluntaria. De esta forma se logra "el efecto transformador, que se obtiene al relacionar el aprendizaje cognitivo de cada participante con la experiencia de todos" (Vargas, 2020, p. 144), y además, la reflexión personal que cada persona orientada va vivenciando con el proceso grupal puede enriquecer la perspectiva de las otras personas participantes del grupo, ya que los procesos grupales ayudan "a toda persona, mediante el aprendizaje social, a dilucidar otras perspectivas, entre ellas clarificarse con respecto a los asuntos individuales; a enfrentar las transiciones o situaciones problema propias del desarrollo y así adaptarse a ello con mayor certeza y facilidad” (Vargas, 2020, p. 136). Las devoluciones que la persona profesional en orientación realiza a las personas que voluntariamente participan en esta parte de la sesión compartiendo sus reflexiones personales, pueden fundamentarse en los pasos del diseño de vida llamados deconstrucción, reconstrucción y co-construcción, explicados anteriormente en este ensayo. Esto permite que se produzca un "reaprendizaje colectivo que favorece en cada participante el autodescubrimiento de su propio crecimiento" (Vargas, 2020, p. 136).

También, en este segundo momento de las sesiones, la persona profesional en orientación puede usar una pedagogía problematizadora para generar un diálogo crítico y emancipador (desde la pedagogía crítica), aprovechando las respuestas que las personas estudiantes comparten y los comentarios que se generen a partir de ellas, para presentar ejemplos de problemas sociales del mundo real, y así involucrar a las personas estudiantes en un diálogo e investigación colaborativos para desafiar la comprensión comúnmente aceptada de los problemas sociales. La intención es facilitar el pasar de los entendimientos individualistas sobre la pobreza y el desempleo (por ejemplo, que estas se dan por la pereza y la falta de persistencia), hacia el reconocimiento de las barreras estructurales de la misma, por ejemplo, la escasez de empleo, los bajos salarios, la falta de beneficios y las prácticas discriminatorias (Kenny et al, 2019). De esta forma se va generando la reflexión crítica que lleva al compromiso con la acción transformadora, según lo plantea la pedagogía crítica antes explicada.

Al mismo tiempo que se va desarrollando la sesión grupal, cada participante del grupo puede ir analizando sus propios trabajos realizados en su portafolio a partir de las narraciones personales escritas y los comentarios que se generan en el dialogo crítico.

En un tercer momento, se invita a que cada participante escriba en su portafolio sus conclusiones personales sobre lo vivido en la sesión. También, la persona profesional en Orientación puede invitar a las personas estudiantes a profundizar en las reflexiones tratadas en esta sesión en los próximos días. Incluso a aquellas personas que sientan que no pudieron encontrar alguna respuesta a las preguntas planteadas, se les puede dejar de tarea voluntaria el realizarla en sus casas. De esta manera, en la siguiente sesión, antes de empezar con el primer momento de la sesión, se puede sacar un pequeño espacio para ver si alguna persona quisiera compartir algo de esta tarea voluntaria o bien de lo que profundizo en los días anteriores.

En resumen, se trata de ayudarles a las personas orientadas a incluir en sus narrativas vocacionales el tema de la causa de justicia social en que la persona quiera involucrarse con su carrera (en el sentido amplio del término). Si a esto le sumamos la visión de servicio, es decir, que sin importar la ocupación que se desempeñe 
siempre puede realizarse como una forma de servirle a la humanidad, haciendo lo mejor posible las tareas propias del trabajo/estudio; esto también puede colaborar en re-significar la ocupación y verla como una parte de la construcción de su propia carrera vocacional.

Durante los tres momentos antes expuestos, es importante que la persona profesional en Orientación acompañe a las personas orientadas para que estas puedan tomar en cuenta el concepto amplio de carrera que maneja Hooley (2021), el cual abarca tanto los trabajos pagados y no pagados, la educación y formación recibidos y otras actividades con propósitos que realizan los individuos, por ejemplo, voluntariados y compromisos familiares. Esto es importante de destacar, debido a que las realidades de los contextos latinoamericanos podrían limitar algunas acciones a llevar a cabo en relación con cursos de formación u ocupaciones laborales a los que la persona podría acceder. Sin embargo, esta visión amplia de carrera puede permitir que la persona tome decisiones vocacionales que ayuden a seguir construyendo su carrera, aunque esta no implique necesariamente un curso universitario o un trabajo específico.

Los voluntariados, los cursos cortos, las autoformaciones valiéndose de los recursos que la internet da y otros compromisos adquiridos a niveles familiares, de iglesia o comunidad en donde se vive, entre otros similares, podrían permitir el alcance de acciones que la persona orientada se plantee para seguir construyendo su carrera a partir de lo vivenciado en el proceso de orientación en el que está participando. Esta visión podría ayudar a conciliar el hecho de que, en muchos casos, las personas no puedan trabajar y/o estudiar en aquello en lo que se sienten llamados (aquello que creen es su vocación) o simplemente en aquello que desearían, debido a diversos factores del contexto sociopolítico, sino que se ven vistos en la necesidad de trabajar o estudiar en lo que pueden desde su realidad, para poder, por ejemplo, llevar los recursos económicos necesarios para subsistir.

Finalmente, y de ser posible, la persona profesional en Orientación podría revisar los portafolios de trabajo de las personas orientadas entre una sesión grupal y la que sigue. Esto le permitiría escribirle algunas devoluciones en el portafolio que la persona orientada podría leer al principio de la siguiente sesión. De esta forma se aprovecha la oportunidad que da el portafolio para que el docente (en este caso la persona profesional en Orientación en cuanto educador) reflexione y comente sobre lo elaborado por el estudiante en el portafolio (Danielson y Abrutyn, 1999).

La persona profesional en Orientación puede también hacer invitaciones específicas a algunas personas que identifique para que compartan algo de lo que escribieron que se considere valioso de llevar al grupo en la siguiente sesión, siempre respetando la voluntariedad de cada participante. Y también, se podría identificar casos de personas orientadas que sean importantes de atender de manera individual. Si este fuera el caso, podría invitar a la persona a recibir un proceso de orientación individual para ampliar algunas de las narraciones presentadas por escrito en el portafolio elaborado en el proceso grupal. Y esto a su vez le daría material de trabajo para empezar el proceso de atención individual con algunas personas específicas.

\section{Conclusiones}

El mundo ha cambiado, y la realidad actual merece que las personas profesionales de la Orientación Vocacional cuenten con herramientas actualizadas que puedan responder a las demandas de las personas orientadas de este siglo, caracterizado por ser un mundo incierto, en donde las carreras de vida no son lineales ni predecibles y son siempre cambiantes. El paradigma del discurso narrativo del diseño de vida brinda herramientas que se pueden aplicar no solamente a las atenciones de orientación vocacional de manera individual, sino que además pueden ser aplicadas en las intervenciones grupales. Los conceptos de 
narrativa de la identidad, flexibilidad y el mantenerse empleable, así como los cinco pasos propuestos en las intervenciones desde el paradigma del diseño de vida y las preguntas propias de la entrevista de construcción de carrera, se pueden rescatar para ser aplicados en intervenciones grupales en contextos latinoamericanos.

Para poder responder a las críticas que se le hacen a la teoría de construcción de carrera, de que responde a una realidad diversa a la latinoamericana, con una lógica de teoría de capital humano que fomenta la individualización como principal característica de las sociales posmodernas, las intervenciones desde este paradigma se pueden complementar con la visión de la pedagogía crítica y emancipadora, la cual promueve una concientización de las diversas realidades sociales mediante el diálogo crítico y problematizador, para, por medio de la vivencia de la propia vocación, aportar en la transformación social necesaria frente a las diversas realidades que necesitan de intervenciones desde la justicia social.

Otra acción importante es la facilitar experiencias culturales que extiendan las narrativas vocacionales de las personas estudiantes, ya que se amplifica la visión que muchas veces se ve limitada por los contextos sociales y familiares en que se desenvuelven.

El uso de la estrategia de portafolio puede ser útil para estos procesos grupales guiados por la teoría de la construcción de carrera y la pedagogía crítica. Además, en los procesos de orientación vocacional puede usarse un concepto amplio de carrera, que no incluya solamente los cursos de formación o trabajos realizados, sean estos pagados o no, sino que además incluya voluntariados y otros compromisos sociales y familiares que la persona desempeña a lo largo de su trayectoria de vida, buscando dentro de ellos los temas recurrentes que conforman su narrativa de la identidad.

\section{Referencias}

Cunti, A. y Priore, A. (2020). Aiutami a scegliere. L'orientamento nella relazione educative. Il mestiere della pedagogia [Ayúdame a elegir. La Orientación en las relaciones educativas. La profesión de pedagogía]. Franco Angeli.

Danielson, C. y Abrutyn, L. (1999). Una introducción al uso de portafolios en el aula. Fondo de Cultura Económica. http://www.terras.edu.ar/biblioteca/3/3EEDU\%20-\%20Danielson\%20-\%20Portafolios\%20-\%20 Unidad\%204.pdf

Freire, P. (1970). Pedagogía del oprimido. Siglo XXI Editores. https://redclade.org/wp-content/uploads/freire.pdf

Freire, P. (2004). La educación como práctica de la libertad. Siglo XXI editores.

Hooley, T. (2021). Career Development and Human Capital Theory: Preaching the "Education Gospel” [Desarrollo de carrera y la teoría de capital humano: predicando el "evangelio de la educación”]. En P. Robertson, T. Hooley y P. McCash (Eds.), The Oxford handbook of career development. Oxford University Press. 10.1093/ oxfordhb/9780190069704.013.5

Hyslop-Margison, E. y Ayaz, M. (2007). Career Education as Humanization: A Freirean Approach to Lifelong learning [La educación de la carrera como humanización: Un enfoque Freireano al aprendizaje para toda la vida]. The Alberta Journal of Educational Research, 53(4), 347-358.

Juntunen, C. L., Motl, T. C. y Rozzi, M. (2019). Major Career Theories: International and Developmental Perspectives [Las principales teorías de carrera: Perspectivas internacionales y de desarrollo]. En J. A. Athanasou y H. N. Perera(Eds.), International Handbook of Career Guidance (pp. 45-72). Springer.https://doi.org/10.1007/978$\underline{3-030-25153-6 \quad 3}$ 
Kenny, M., Blustein, D., Liang, B., Klein, T. y Etchie, Q. (2019). Applying the Psychology of Working Theory for Transformative Career Education [Aplicando la teoría de la psicología del trabajo a la educación de carrera transformadora] Journal of Career Development, 46(6), 623-636. https://doi. org/10.1177\%2F0894845319827655

McMahon, M. (2014). New trends in theory development in career psychology [Nuevas tendencias en teorías del desarrollo dentro de la psicología de las carreras]. En G. Arulmani, A. J. Bakshi, F. T. L. Leong y A. G. Watts (Eds.), Handbook of career development: International perspectives (pp. 13-27). Springer. https://doi. org/10.1007/978-1-4614-9460-7_2

McMahon, M. y Arthur, N. (2019). Career development theory, origins and history [Teorías de desarrollo de carrera, sus orígenes e historia]. En N. Arthur y M. McMahon. (Eds.), Contemporary theories of career development. (pp. 241-257). Routledge. https://doi.org/10.4324/9781315276175

Puertas, A. (2014, 1-3 octubre). Paradigmas y prácticas para la intervención de la carrera en el siglo XXI (Basado en The Theory and Practice of Career Construction de Mark L. Savickas). En Colegio de Profesionales en Orientadción (Ed.), Memoria Primer Congreso del Colegio de Profesionales en Orientación "50 años de la Orientación en Costa Rica" (pp. 35-66). Autor.

Savickas, M. L., Nota, L., Rossier, J., Dauwalder, J.-P., Duarte, M. E., Guichard, J., Soresi, S., Van Esbroeck, R. y Van Vianen, A. (2009). Life designing: A paradigm for career construction in the 21st century [Diseño de vida: Un paradigma para la construcción de carrera en el siglo 21]. Journal of Vocational Behavior, 75 (3), 239-250. https://doi.org/10.1016/j.jvb.2009.04.004

Savickas, M. L. (2012). Life Design: A paradigm for career intervention in the $21^{\text {st }}$ century [Diseño de vida: un paradigma para las intervenciones de carrera en el siglo 21]. Journal of Counseling \& Development, 90(3), 1319. https://doi.org/10.1111/j.1556-6676.2012.00002.x

Savickas M. L. (2013). Career Construction Theory and Practice [Teoría y práctica de construcción de carrera]. En S. Brown y R. Lent (Eds.), Career Development and Counseling. Putting theory and research to work (2. ${ }^{a}$ ed., pp. 147-186). John Wiley \& Sons.

Savickas, M. L. (2015). Life-Design Counseling Manual [Manual de Orientación con el diseño de vida]. http://www. vocopher.com/LifeDesign/LifeDesign.pdf

Savickas, M. L., Savickas ,S. (2019). A History of Career Counselling [Una historia de Orientación para la carrera]. En J. A. Athanasou, y Perera, H. N. (Eds.), International Handbook of Career Guidance. (pp. 25-43). Springer. https://doi.org/10.1007/978-3-030-25153-6_2

Vargas, Z. R. (2020). La Orientación: Una revisión histórico - metodológica. EdiNExo. 